\title{
PECULIAR CO DISTRIBUTION IN ACTIVE SPIRAL GALAXIES: NCG4258 AND CIRCINUS
}

\author{
M. KRAUSE \\ Max-Planck-Institut für Radioastronomie \\ Auf dem Hügel 69, D-53121 Bonn, Germany \\ N. NEININGER \\ Radioastronomisches Institut der Universität Bonn \\ Auf dem Hügel 71, D-53121 Bonn, Germany \\ M. ELMOUTTIE AND K.L. JONES \\ The Physics Department of the University of Queensland \\ QLD4072, Australia \\ AND \\ R.F. HAYNES \\ The Australia Telescope National Facility \\ CSIRO, P.O. Box 76, NSW 2121, Australia
}

\section{Introduction}

NGC4258 and Circinus are both nearby spiral galaxies with active galactic nuclei which show high velocity water maser emission and Seyfert 2 characteristics. Both show radio emission perpendicular to the normal spiral arms which seem to be related to the nuclear activity (van der Kruit et al., 1972; Harnett et al., 1990). In NGC 4258 they coincide with the inner part of the rapidly rotating nuclear disk as expected for nuclear jets. Due to the strong misalignment of the nuclear disk plane with the galaxy's disk plane the jets pass the galactic disk. Molecular gas in NGC 4258 has only been detected in the nucleus and along the $\mathrm{H} \alpha$ jets up to a distance of $2 \mathrm{kpc}$ from the nucleus (Krause et al., 1990).

\section{Observations}

We observed NGC 4258 in ${ }^{12} \mathrm{CO}(1-0)$ with the Plateau de Bure interferometer (PdBI) in its compact configuration in 5 fields along the $\mathrm{H} \alpha$ jets using 
the mosaicing technique. In total we observed an area of $100^{\prime \prime} \times 40^{\prime \prime}$, with a uniform sensitivity along the major axis of the oval. In order to correct the data for 'missing spacings', we combined the PdB data in the UV plane with single dish data obtained recently with the $30-\mathrm{m}$ telescope at Pico Veleta by Cox and Downes (1997). The angular resolution of the final maps is 4 "' $6 \times 33^{\prime \prime} 3$.

The Circinus galaxy was observed at the Swedish ESO Submillimeter Telescope (SEST) in ${ }^{12} \mathrm{CO}(1-0)$ along a rectangular grid pattern centered at the galaxy's optical core along and perpendicular to the radio major axis with a cell size of $20^{\prime \prime}$. The angular resolution of the data is $45^{\prime \prime}$.

\section{Discussion}

The $\mathrm{CO}$ in $N G C 4258$ follows two ridges along the $\mathrm{H} \alpha$ emission with a total extent of $100^{\prime \prime}(3 \mathrm{kpc})$. The width of each ridge is about $10^{\prime \prime}$. They are symmetrically located relative to the jet axis with a region of minor $\mathrm{CO}$ emission in between. Contrary to previous interpretations the $\mathrm{CO}$ does not outline 'walls' around the $\mathrm{H} \alpha$ jets and collimates it, but rather it seems to be mixed (in location and in velocity) with the $\mathrm{H} \alpha$ gas. Whereas most of the $\mathrm{CO}$ gas takes part in the normal disk rotation, there is a steep velocity gradient of about $300 \mathrm{~km} / \mathrm{s}$ along the jet axis. This strongly suggests that the $C O$ gas is rotating around the jet axis. The sense of rotation is equal in the northern and southern jet and equal to that of the rapidly rotating nuclear disk and the helical structure in $\mathrm{H} \alpha$ as found by Cecil et al. (1992). Hence, our observations support the hypothesis that the molecular gas (and probably also the $\mathrm{H} \alpha$ gas) is entrained disk material which has been partly ionized by shocks and which is rotating around the jet axis along helices.

We have detected strong $\mathrm{CO}$ emission in Circinus larger in extent than the radio emission but much smaller than the HI phase. It is concentrated towards the nuclear region. Our data suggest the presence of a central molecular disk or ring with a radius of $300 \pm 50 \mathrm{pc}$ rotating with a velocity of about $200 \mathrm{~km} / \mathrm{s}$ around a central source which is responsible for a bipolar outflow. The integrated CO map shows a spur along the northern minor axis, possibly related to the outflow.

\section{References}

Cecil G., Wilson A.S., Tully R.B. (1992) ApJ, 390, 365

Cox P., Downes D. (1996), ApJ, 473, 219

Harnett J.I., Whiteoak J.B., Reynolds J.E., Gardner F.F., Tzioumis A., 1990, MNRAS, 244, 130

Krause M., Cox P., Garcia-Barreto J. A., Downes D. (1990) $A \& A$, 233, L1

Van der Kruit P.C., Oort J.H., Mathewson D.S. (1972), $A \& A$ 21, 169 